\title{
Étude des spectres de houle à proximité des côtes
}

\section{Wave spectra in the vicinity of the coast}

\author{
PAR M. GOHIN
}

ingtinteur a la sogreah, Lievtenant de valsseau (c.h.)

\author{
Communication présentée à la Société Hydrotechnique de France le 18 juin 1959
}

\begin{abstract}
La méthode de Tukey permet d'obtenir à partir d'un enregistrement de la dénivellation en fonction du temps le spectre d'énergie de la houle. Les spectres d'énergie obtenus en un même point d'observation se classent en un certain nombre de familles: les spectres d'une famille ont tous la même forme.

pour pouvoir prévoir les spectres de la houle, il faut avoir, en particulier, étudié les relations entre les paramètres fondamentaux des spectres et les données météorologiques. Lorsque la prévision sera suffisamment sûre, on pourra énvisager d'établir la statistique des spectres d'énergie.
\end{abstract}

\begin{abstract}
Tukey's method makes it possible to oblain wave energy spectra from a recording of water level changes with time.

The energy spectra obtained at a given obserb. ation point can be classified in families. All the spectra of any one family have the samo shape.

In urder to be able to forecast wane spectra il is necessary to have studied the relationships between the fundamental paramelers of the spectra and of the corresponding meteorological data. When forecasts become sufficienlly accurate il will be possible to prepare energy spectra statistics.
\end{abstract}

\section{AVANT-PROPOS}

L'ćtude de la houle retient depuis une cinquantaine d'années l'attention de nombreux mathématiciens et physiciens; la prévision de la houle a été tout particulièrement développée depuis une vingtaine d'années. Les méthodes proposées sont en général valables en pleine mer, c'est-à-dire par grand fond et à grande distance des côles; certains auteurs ont étudié la prévision de la houle pour une région côtière déterminée - par exemple Gelci et Cazalet pour le Maroc.
Dans cet article, on présente une méthode d'étude faisant largement appel à l'expérience. Elle s'applique à toutes les régions côtières, et conduit non seulement à la prévision de la houle, mais aussi à la prévision de la statistique des efforts de la houle sur un ouvrage maritime.

La pluparl des figures du lexte proviennent de dépouillements de relevés effectués au sud des iles Chausey par le Service d'Etudes sur l'utilisation des marées de l'Electricité de France.

\section{I. - DÉPOUILLEMENT D'ENREGISTREMENT}

Considérons un navire immobile situé en une position $\mathrm{N}$ de la mer; à bord de ce bâtiment, un sondeur très précis enregistre la profondeur $z$ en fonction du temps $t$.

Examinons cette courbe $z(t)$; les ordonnées fluctuent rapidement aulour d'une certaine valeur moyenne $Z\left(^{*}\right)$; ces fluctuations sont dues

(*) $Z$ varie au cours du temps en raison de la marée; sa valeur moyenne $h$ est la profondeur au lieu $N$. 
a la houle. Nous appellerons « dénivellations» et noterons $\zeta$ la différence : $z-Z$ et nous admettrons dans tout ce qui suit que l'enregistrement effectué est celui de la dénivellation au cours du temps $\zeta(t)$.

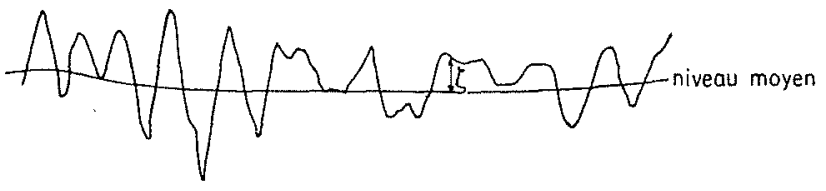

FIG. 1

Etudions la portion de la courbe $\zeta(t)$ enregistrée sur une durée $\theta$ de quelques minutes, à partir d'un instant arbitrairement choisi $t_{i}$. L'analyse harmonique classique de Fourier permet d'obtenir un spectre de raies. La période $\mathbf{T}_{m}$ de la raie d'ordre $m$ est $\theta / m$, son amplitude complexe $\overline{\mathrm{A}}_{m}$ est donnée par la relation :

$$
\overline{\mathrm{A}}_{m}=\frac{1}{0} \int_{t_{i}}^{t_{i}+\theta} \zeta(t) e^{-j} \frac{2 \pi m t}{\theta} d t
$$

Inversement, la dénivellation $\zeta$ à un instant $t$ compris entre $t_{i}$ et $t_{i}+\theta$ est donnée par la relation :

$$
\zeta=\sum_{m=-\infty}^{m=+\infty} \bar{A}_{m} e^{j 2 \pi(m t / \theta)}
$$

Admettons que les conditions météorologiques (vitesse du vent, pression, ete.) et océanographiques (profondeurs, courants de marée, etc.) ne varient pas d'une position à l'autre de la surface de la mer visible depuis le navire, et ne varient pas non plus dans l'heure qui suit l'instant $t_{i}\left(^{\star}\right)$.

A partir d'un nouvel instant initial $t^{\prime}$, effectuons le déponillement de $\zeta^{\prime}(t)$ enregistrée sur Ia même durée : les $\overline{\mathrm{A}}_{m}^{\prime}$ obtenus sont différents $\operatorname{des} \overline{\mathbf{A}}_{m}$.

Imaginons qu'un autre navire est situé en $\mathrm{N}_{1}$ - en decà de l'horizon visible depuis $\mathrm{N}$ - et enregistre la dénivellation $\zeta_{1}(t)$ à partir de $t_{i}$ sur la durée 0 . L'analyse conduit à un spectre de raies dont les amplitudes $\bar{A}_{m 1}$ sont différentes de $\overline{\mathbf{A}}_{m}$.

En d'autres termes, bien que grosso modo les spectres de $\zeta(t), \zeta^{\prime}(t)$ et $\zeta_{1}(t)$ soient semblables, il n'en reste pas moins que, pour des enregistrements ayant par hypothèse les mêmes pro-

(*) Ces précisions sur la stationnarité de la houle dans le temps et l'espace étant données là simplement pour fixer les id̉ées. priétés statistiques, dans te détail ces spectres sont différents.

On dispose depuis quelques années d'une nouvelle méthode, due à Tukey, permettant d'obtenir, pour des surfaces de la mer ayant les mêmes propriétés statistiques, un même spectre. Nous décrirons dans cette première partie les étapes de cette méthode $\left(^{*}\right)$.

En premier lieu, on détermine, nous y reviendrons, la valeur précise des paramètres suivants :

0 durée de l'enregistrement : au cours de cette durée, la houle est considérée comme stationnaire. Ce caractère est absolument essentiel.

$2 \Delta t$ et $2 \mathrm{~m} \Delta t$ périodes minimum et maximum présentant quelque importance.

En second lieu, on divise $\theta$ en $\mathbf{N}$ intervalles élémentaires de durée $\Delta t$. Pour chaque valeur ist du temps, compté depuis l'origine de la portion d'enregistrement retenue, on relève la dénivellation $\zeta_{i}$.

On calcule ensuite la «fonction » d'autocolrélation suivante :

$$
\mathrm{R}_{q}=\frac{1}{\mathrm{~N}-q} \sum_{i=1}^{i=x-q} \zeta_{i} \zeta_{i+q}\left(^{\star \star}\right)
$$

potur $q=0,1, \ldots, m-1, m$.

On porte sur un graphique les valeurs $\mathbf{R}_{q}$ en fonction des abscisses $q \Delta t$. La courbe interpolant les points $R_{t}$, courbe dite « correlogramme», est la suivante :

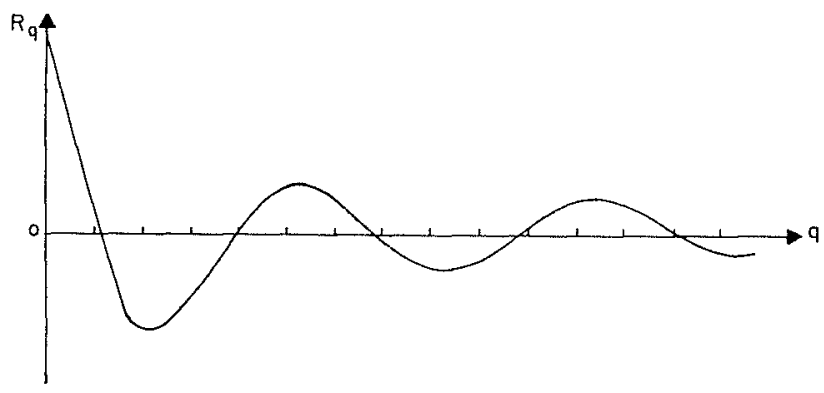

FIG. 2

Ia « fonction » $R_{q}$ est paire. En effet, au lieu de multiplier la dénivellation au temps ist par la dénivellation au temps postérieur $(i+q) \Delta t$, on pourrait tout aussi bien multiplier la dénivellation au temps $j \Delta t$ par la dénivellation au

(*) Référence : Article paru dans « Symposium on applications of autocorrelation analysis to physical problems », Office of Naval Research Department of the Navy, June 1949.

$\left({ }^{* *}\right)$ Cette «fonction» est en fait une série de valeurs discrètes. 
temps antérieur $(j-q) \Delta t$. On obtiendrait la «onction»:

$$
\mathrm{R}_{-q}=\frac{1}{\mathrm{~N}-q} \sum_{j=\mathrm{N}}^{j=1+q} \zeta_{j} \zeta_{j-q}
$$

En posant $j-q=i$, il vient :

d'où : $\mathbf{R}_{-q}=\mathbf{R}_{q}$.

$$
\mathrm{R}_{-q}=\frac{1}{\mathrm{~N}-q} \sum_{i=\mathrm{N}-q}^{i=1} \zeta_{i+q} \zeta_{i}
$$

En troisième lieu, on procède à l'analyse harmonique de Fourier de la «fonction » $\mathbf{R}_{q}$ sur l'intervalle $2 \mathrm{m \Delta t}$. Cette analyse ne comporte que des termes en cosinus, puisque $\mathrm{R}_{-q}=\mathrm{R}_{q}$.

La raie d'ordre $p$ est donnée par l'expression :

$$
\mathrm{E}_{p}=\frac{1}{m} \mathrm{R}_{q}+\frac{2}{m} \sum_{q=1}^{q=m-1} \mathrm{R}_{q} \cos -\frac{p q \pi}{m}+\frac{1}{m} \mathrm{R}_{m} \cos p \pi
$$

$\mathrm{E}_{p}$ représente, à un facteur de proportionnalité constant près, l'énergie contenue dans la bande de fréquence,

$$
\left.\frac{2 \pi}{2 m \Delta t}\left(p-\frac{1}{2}\right) \leqslant k \leqslant \frac{2 \pi}{2 m \Delta t}: p+\frac{1}{2}\right)
$$

centrée sur la fréquence :

$$
k_{p}=\frac{2 \pi p}{2 m \Delta t}
$$

Ce spectre est dit «spectre d'énergie», nous le noterons $\left(\mathrm{E}_{p}, k_{p}\right)$.

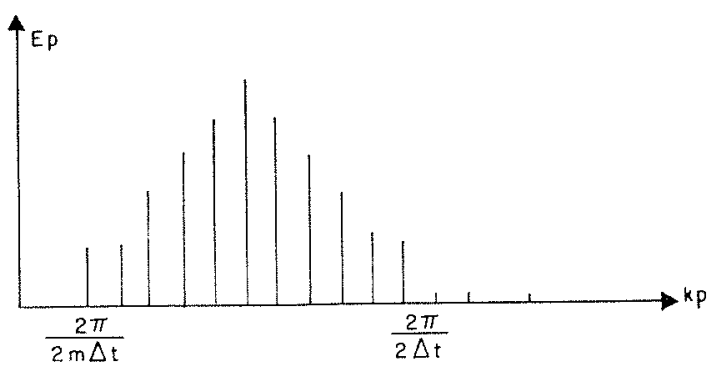

Frg. 3

En pratique $\left(\mathrm{E}_{p}, k_{p}\right)$ s'étend de :

$$
k_{p}=\frac{2 \pi}{2 \mathrm{~m} \Delta t} \text { aे } k_{p}=\frac{2 \pi}{2 \Delta t}
$$

Examinons maintenant le choix des paramètres $\theta, 2 \Delta t, m$.

1. La durée $\theta$ est fixée dans une certaine mesure par le caractère stationnaire du phénomène enregistré. Pour la houle, on pourra prendre : $\theta=10$ minutes pour une houle s'établissant, $0=30$ minutes pour une mer bien formée.
2. La plus petite période $2 \Delta t$ dépend du phénumène étudié; on la fixe par expérience à une valeur de l'ordre de quelques secondes.

3. Le choix de $m$, ou encore de $2 m \Delta t$, peut être fait en considérant un ou plusieurs des points suivants :

a) Le nombre de raies désiré : $m$.

b) La séparation de deux périodes $\mathrm{T}_{1}$ et $\mathrm{T}_{2}$ :

$$
\frac{2 \pi}{\mathrm{T}_{1}}-\frac{2 \pi}{\mathrm{T}_{2}}=\frac{2 \pi}{2 m \Delta t}
$$

d'oì :

$$
2 m \Delta t=\frac{\mathrm{T}_{1} \mathrm{~T}_{2}}{\mathrm{~T}_{2}-\mathrm{T}_{1}}
$$

c) La précision $\varepsilon$ du spectre étroitement liée au rapport :

$$
\frac{0}{2 m \Delta t}
$$
tant.

Examinons ce point particulièrement impor-

Si l'on pouvait prendre 0 très grand, la méthode de Tukey conduirait au spectre d'énergie $\operatorname{exact}\left(\mathbf{E}_{p}^{*}, k_{p}\right)$.

0 étant limité, le calcul fournit le spectre $\left(\mathrm{E}_{p}, k_{p}\right)$.

Nous appellerons précision et noterons $\varepsilon$, chiffré en \%, un nombre tel que l'on ait la double inégalité :

$$
\frac{\varepsilon \mathrm{E}_{p}}{100} \leqslant \mathrm{E}^{*}{ }_{p} \leqslant \frac{100}{\varepsilon} \mathrm{E}_{p}
$$

avec une probabilité de $90 \%$; autrement dil, l'on a :

- 5 chances sur 100 pour que la valeur vraic $\mathrm{E}^{*}{ }_{p}$ soit inférieure à $(\varepsilon / 100) \mathrm{E}_{p}$.

- 5 chances sur 100 pour que la valeur vraie $\mathrm{E}^{*}{ }_{p}$ soit supérieure à $(100 / \varepsilon) \mathrm{E}_{p}$.

Tukey a établi, moyennant cerlaines hypothèses restrictives, une loi donnant $\varepsilon$; en simplifiant cette loi, on peut admeltre que $\varepsilon$ est lic au rapport $0 /(2 m \Delta t)$, pour les valeurs de $0 /(2 m \Delta t)$ les plus courantes, par l'expression :

$$
\varepsilon=85-300 \times \frac{2 m \Delta l}{0}
$$

et on a souvent en pratique $\varepsilon$ de l'ordre de 60 à 80 .

Pour chaque valeur $k_{p}$ de la fréquence, on doit associer à $\mathrm{E}_{p}$ un «segment d'incertitude » placé entre les ordonnées :

$$
\frac{\varepsilon \mathrm{E}_{p}}{100} \quad \text { et } \quad \frac{1.00 \mathrm{E}_{p}}{\varepsilon}
$$

(*) Nous noterons par un astérisque les grandeurs exactes, d'ailleurs inaccessibles par le calcul. 
Fn joignant d'une part les ordonnées maxima $100 \mathrm{E}_{p} / \varepsilon$, d'autre part les ordonnées minima $\varepsilon \mathrm{E}_{p} / 100$, on délimite une zone : nous l'appellerons « zone d'incertitude» et la noterons $\left(\mathrm{E}_{p}, \varepsilon\right)$. Nous sommes «quasi-certains» $\left(^{\star}\right)$ que le spectre d'énergie vrai $\left(\mathrm{E}^{*}{ }_{p}, k_{p}\right)$ est à l'intérieur de la zone d'incertitude.

A partir du spectre d'énergie, on peut obtenir diverses grandeurs physiques, dont nous donnerons d'abord les définitions.

Considérons une portion très grossie de la courbe $\zeta(t)$ qui coupe le « niveau moyen » $(\zeta=0)$ par valeurs croissantes aux instants $t$ et $t+\mathrm{T}_{j}$.

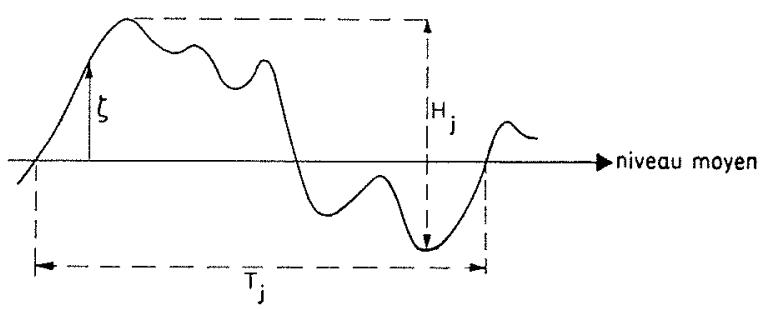

FIG. 4

Nous appellerons « $\mathrm{T}_{j} »$ la période apparente (variable évidemment avec le temps $t$ ); autrement dit, les passages successifs de $\zeta(t)$ par la valeur zéro - la dérivée première étant posilive - se produisent aux instants :

$$
\begin{aligned}
& t_{1}, \quad t_{1}+\mathrm{T}_{1}, \quad t_{1}+\mathrm{T}_{1}+\mathrm{T}_{2}, \ldots \\
& t_{1}+\sum_{1}^{j-1} \mathrm{~T}_{1}, \quad t_{1}+\sum_{1}^{j} \mathrm{~T}_{i}, .
\end{aligned}
$$

Entre $t$ et $t+\mathrm{T}_{j}$, $\zeta$ passe par un maximum maximorum et un minimum minimorum; on notera $H_{j}$ la différence algébrique de ces deux extrêmes, $\mathrm{H}_{j}$ est la hauteur apparente.

On appellera « hauteur moyenne apparente mesurée » $\overline{\mathrm{H}}$ la valeur moyenne de tous les $\mathrm{H}_{j}$, et «période moyenne apparente mesurće 》 la valeur moyenne de tous les $T_{j}$.

Rice et Longuet-Higgins $\left(^{*}\right)$ ont établi, pour un spectre continu et suffisamment étroit, des expressions donnant les valeurs : de l'énergie totale, de la hauteur moyenne apparente calculée, de la période moyenne apparente calculée.

Dans le cas d'un spectre de raies, ces formules sont les suivantes:

- Energie : $\mathrm{E}=\underset{p}{\Sigma} \mathrm{E}_{p}$.

- Hauteur : $\overline{\mathrm{H}}_{c}=\sqrt{\pi \mathrm{E}}$.

- Période :

$$
\overline{\mathrm{T}}_{c}=2 \pi \sqrt{\frac{\mathrm{E}}{\Sigma k_{p}{ }^{2} \mathrm{E}_{p}}}=2 m \Delta t \sqrt{\frac{\mathrm{E}}{\sum_{p} p^{2} \mathrm{E}_{p}}}
$$

L'expérience montre que l'on obtient en général, c'est-à-dire lorsque les conditions de validité de ces formules sont satisfaisantes:

$$
\overline{\mathrm{H}}_{i} \not t \overline{\mathrm{H}} \quad \overline{\mathrm{T}}_{t} \# \overline{\mathrm{T}}
$$

les erreurs étant de l'ordre de quelques ot.

\section{II. - FAMILLES DE SPECTRES. - SPECTRES TYPES}

Admettons que, pour un lieu d'observation donné, nous ayons réuni une collection de spectres d'énergie. Nous nous proposons, par une nouvelle étude, de classer ces spectres logiquement.

Reprenons le spectre $\left(\mathrm{E}_{p}, k_{p}\right)$; d'après la définition même de $\mathrm{E}_{p}$, l'amplitude des raies est proportionnelle à la largeur de bande:

$$
\Delta k=\frac{2 \pi}{2 m \Delta t}
$$

Il est donc tout indiqué de s'affranchir, dans le tracé, du paramètre de dépouillement $2 \mathrm{m \Delta}$

(*) Cette expression rappelle que l'on a 10 chances sur 100 seulement de voir $\mathrm{E}^{*}$, sortir de la zone d'incertitude. en représentant le spectre sous la forme du «spectre de densitć spectrale»:

$$
\left(\mathrm{W}_{p}=\frac{\mathrm{E}_{p}}{\Delta k}, k_{p}\right)
$$

Si $\Delta k \rightarrow 0, \mathrm{~W}_{p} \rightarrow \mathrm{W}$ fonction continue de la fréquence appelée « courbe de densité spectrale énergétique »W( $k)$.

Considérons d'une part une valeur particulière $k_{1}$ de la fréquence, d'autre part une valeur particulière $W_{1}$ de la densité spectrale [non obligatoirement prise sur la courbe $W(k)]$.

$\left(^{\star}\right)$ Cf. en particulier « The statistical analysis of a random, moving surface » par Longuer-Higgins, publié dans les « Philosophical transactions of the Royal Society of London $\gg$, Series A, Mathematical and Physical Sciences, volume 249 , pp. 321 à $387(21-2-57)$. 
Posons :

$$
y=\frac{\mathrm{W}}{\mathrm{W}_{1}} \quad x=\left(\frac{k}{k_{1}}\right)^{-1}
$$

on a :

$$
\begin{gathered}
\int_{0}^{\infty} \mathrm{W} d k=\int_{0}^{\infty}-y \mathrm{~W}_{1} x^{-2} k_{1} d x \\
=\mathrm{W}_{1} k_{1} \int_{0}^{\infty} x^{-2} y d x=m_{0} \mathrm{~W}_{1} k_{1} \\
\operatorname{avec} m_{0}=\int_{0}^{\infty} x^{-2} y d x \\
\int_{10}^{\infty} k^{2} \mathrm{~W} d k=m_{2} \mathrm{~W}_{1} k_{1}^{3} \quad \text { avec } m_{2}=\int_{0}^{\infty} x^{-4} y d x
\end{gathered}
$$

Par suite, les relations de Rice s'écrivent:

$$
\begin{aligned}
& \overline{\mathrm{H}}_{c}=\sqrt{\pi} \mathrm{W}_{1}{ }^{1 / 2} \quad k_{1}{ }^{1 / 2} \quad m_{0}{ }^{\mathrm{t} / 2} \\
& \overline{\mathrm{T}}_{\mathrm{c}}=2 \pi k_{1}{ }^{-1} \quad m_{0}{ }^{1 / 2} \quad m_{L^{2}}{ }^{-1 / 2}
\end{aligned}
$$

Inversement :

$$
\begin{aligned}
\mathrm{W}_{1} & =\left(\frac{\overline{\mathrm{H}}_{c}^{2} \overline{\mathrm{T}}_{c}}{2 \pi}\right) \times(1 / \pi) \quad m_{2^{1 / 2}} m_{0}^{-3 / 2} \\
k_{1} & =\left(\frac{2 \pi}{\overline{\mathrm{T}}_{c}}\right) \times m_{0}{ }^{1 / 2} m_{2}^{-1 / 2}
\end{aligned}
$$

Nous prendrons pour valeurs $k_{1}$ et $\mathrm{W}_{1}$ :

$$
\begin{aligned}
& k_{r}=\frac{2 \pi}{\overline{\mathrm{T}}} \\
\text { et : } & \mathrm{W}_{r}=\frac{\overline{\mathrm{H}}^{2} \overline{\mathrm{T}}}{2 \pi}
\end{aligned}
$$

En principe, ces deux valeurs peuvent être oblenues très simplement à partir de mesures faites sur l'enregistrement $\zeta(t)$.

La raie d'ordre $p$ : de fréquence $k_{p}$, d'amplitude $W_{p}$, deviendra la raie réduite d'ordre $p$ :

$$
\begin{aligned}
& \text { - d'abscisse } x_{p}=\frac{k_{r}}{k_{p}}=\frac{2 \pi}{k_{p} \overline{\mathrm{T}}} \\
& \text { - d'ordonnée } y_{p}=\frac{\mathrm{W}_{p}}{\mathrm{~W}_{r}}=\frac{2 \pi \cdot \mathrm{W}_{n}}{\overline{\mathrm{H}}^{2} \overline{\mathrm{T}}^{\mathrm{T}}}
\end{aligned}
$$

La précision du spectre réduit $\left(y_{p}, x\right)$ reste $\varepsilon$. En fait, ceci n'est pas absolument exact, car les mesures faites sur l'enregistrement ne conduisent pas aux vraies valeurs $\overline{\mathrm{H}}^{*}$ et $\overline{\mathrm{T}}^{*}$, mais à des valeurs entachées d'erreur $\overline{\mathrm{H}}$ et $\overline{\mathrm{T}}$. La nouvelle zone d'incertitude $\left(y_{p}, \varepsilon\right)$ contient « fort probablement » la courbe de densité spectrale réduite exacte $y^{*}(\boldsymbol{x})\left(^{\star}\right)$ que nous supposerons être analytique et comporter un nombre minimum de zéros pour toutes ses dérivées.
En effectuant cette réduction sur la totalité des spectres de la collection, on obtiendra pour une suite de valeurs discrètes de $x$ une série de «segments d'incertitude». Pour $x=x_{p}$, ces segmenls se siluent entre :

$$
y_{p i} \times(\varepsilon / 100) \text { et } y_{p i} \times(\mathbf{1 0 0 / \varepsilon}),
$$

$i$ étant le numéro d'ordre du spectre de la collection.

Nous appellerons "famille» un ensemble de spectres dont pour toute valeur de $x$ comprise entre deux limites - arbitrairement fixées, mais identiques pour toutes les familles, par exemple $x=0,5$ et $x=2,5$ - les zones d'incertitude ont une partie commune.

Nous appellerons « spectre type »! $]^{*}$ s de la famille $\Sigma$, la courbe continue et régulière passant au centre de cette partie commune, et nous admettrons que cette courbe est la courbe de densité spectrale réduite vraie de la famille.

En tenant comple des conditions d'établissement du spectre d'énergie, des hypothèses admises par Rice, nous préciserons les limites du tracé du spectre type.

\section{Exemple:}

A partir d'une douzaine d'enregistrements effectués à Chausey, correspondant à des périodes moyennes variables dans le rapport 1 à 3 , et à des hauteurs moyennes variables dans le rapport 1 à 5 , dépouillés dans des conditions variables, on a obtenu une famille caractérisée par le spectre type $y^{*}(x)$, défini pour $0,6 \leqslant x \leqslant 2,1$ tracé sur la figure 5 :

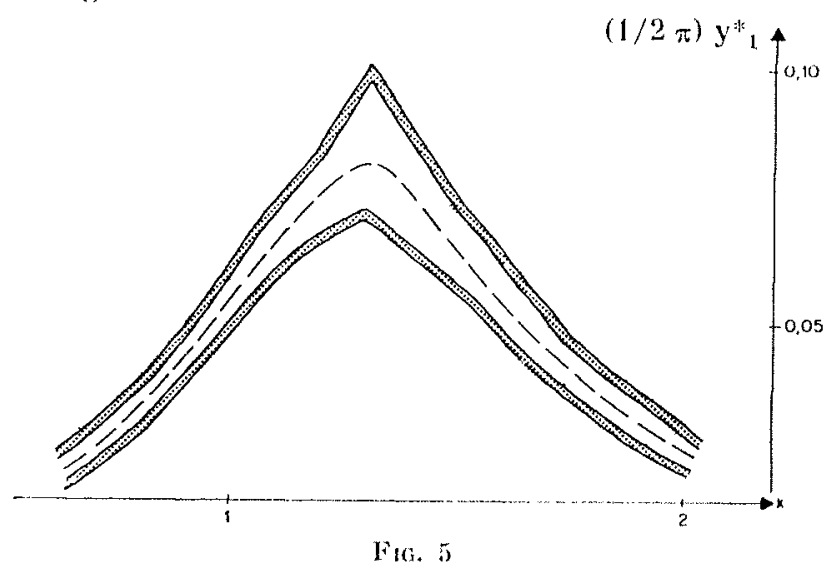

Il est bien clair que ce classement de la collection des spectres en un certain nombre de familles doit constituer un « outil » pratique,

(*) Cette courbe $y^{*}(x)$ est celle qui serait obtenue à partir d'une part de la coube exacte de densite spectrale $W *(k)$, d'autre part des grandeurs de réduction exactes $2 \pi / \overline{\mathrm{T}} *$ et $\mathrm{H}^{*} \mathrm{~T}^{*} / 2 \pi$. 
maniable pour la suite de l'étude de la houle au lieu d'observation. Pour qu'il en soit ainsi, il faut que le nombre de familles soit aussi réduit que possible.

Admettons qu'un premier classement nous ait conduit à ranger $90 \%$ des spectres dans deux familles, toutes deux à une bosse. Nous assouplirons le critère de constitution de la famille - recouvrement des zones d'incertitudes des spectres la formant - pour faire entrer les $10 \%$ restant dans les deux familles.

a) On adjoindra à la partie commune des zones dincertitudes deux bandes latérales dont la largeur sera, par exemple, les $25 \%$ de la largeur de la partie commune (ceci revient en quelque sorte à considérer que l'erreur relative de détermination des $W_{r}$ et $k_{r}$ est de $\pm 25 \%$ ).

b) On introduira la notion de superposition de spectres de houle: dans un spcctre à deux bosses, ou dans un spectre à une bosse, mais à front doux on tentera de distinguer deux spectres élémentaires.

\section{EXEMPLE :}

Le dépouillement d'un enregistrement de Chausey a permis d'établir la zone d'incertitude $\left(W_{p}, \varepsilon\right)$ de la figure. La courbe obtenue à l'aide de $y_{1}^{*}(x)-$ obtenue à Chausey - $\overline{\mathrm{H}}=1,0 \mathrm{~m}$; $\overline{\mathrm{T}}=3,5 \mathrm{~s}$, ne s'inscrit pas dans $\left(\mathrm{W}_{n}, \varepsilon\right)$ pour les grandes périodes.

On introduit alors les deux courbes de densité spectrale :

$$
\begin{aligned}
y^{*}{ }_{1}, \overline{\mathrm{H}}_{1} & =1,0 \mathrm{~m} & \overline{\mathrm{T}}_{1}=3,5 \mathrm{~s} \\
y^{*}{ }_{2}, \overline{\mathrm{H}}_{2} & =0,35 \mathrm{~m} & \overline{\mathrm{T}}_{2}=7,0 \mathrm{~s}
\end{aligned}
$$

La courbe $\mathrm{W}=\mathrm{W}_{1}(k)+\mathrm{W}_{2}(k)$ s'inserit à l'intérieur de $\left(W_{p}, \varepsilon\right)$.

Cette décomposition suppose évidemment qu'un critère permette de distinguer dans les couples $\left(\mathrm{H}_{j}, \mathrm{~T}_{j}\right)$ deux groupements, le premier domnant $\left(\overline{\mathrm{H}}_{1}, \overline{\mathrm{T}}_{1}\right)$, le second $\left(\overline{\mathrm{H}}_{2}, \overline{\mathrm{T}}_{2}\right)$. Nous n'étudierons pas dans cet article ce critère.

\section{REMARQUE :}

\section{Spectre type de Neumann.}

Pierson et Neuman (*) ont établi, pour la mer « pleinement développéc », la formule suivante :

$$
\mathrm{W}^{*}=\mathrm{A} k^{-6} e^{-b k^{-2}}
$$

(*) Cf. par exemple « Ships at Sea », "The Seaway», par Korvin KRoukovskr, janvier 1957.

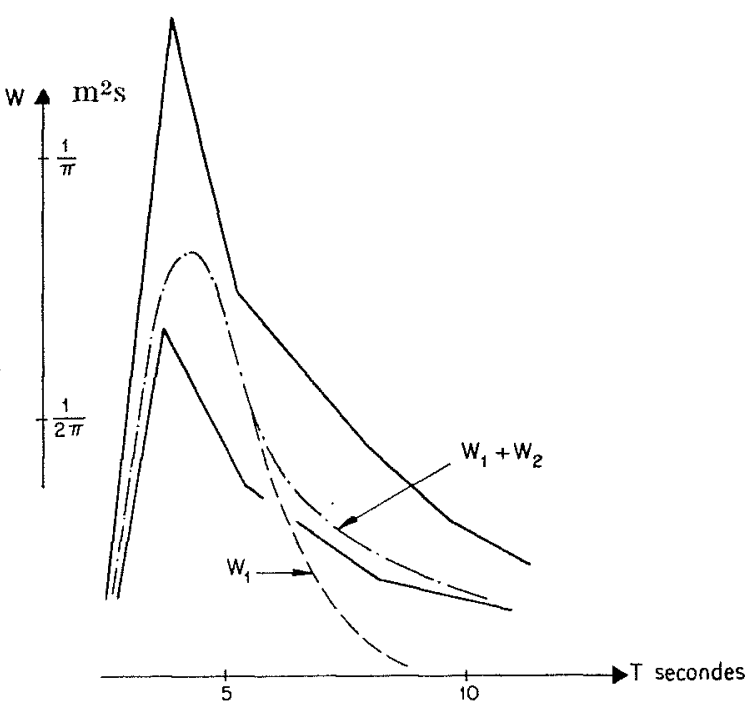

Fia. 6

où A est une constante et $b$ un coefficient du type $b=\beta / v^{2}$, $v$ étant la vitesse du vent.

La courbe de densité spectrale présente un maximum pour :

$$
\frac{d \mathrm{~W}}{d k}=0 \quad \text { soit pour } k=k_{\mathrm{M}}=\sqrt{b / 3} .
$$

On prendra pour réduire le spectre $k_{\mathrm{M}}$ et $\mathrm{W}_{\mathrm{M}}$ :

$$
\frac{\mathrm{W}}{\mathrm{W}_{\mathrm{M}}}=\left(k_{\mathrm{MI}} / k\right)^{6} e^{3}\left[1-\left(k_{\mathrm{M}} / \boldsymbol{k}\right)^{2}\right]
$$

On obtient, en utilisant la relation :

$$
\begin{gathered}
\int_{0}^{\infty} x^{m} e^{-n^{2}} d x=(1 / 2) n-(m+1) / 2 \Gamma[(m+1) / 2] \\
W^{*}{ }_{M}=0,53 \frac{\overline{\mathrm{H}}^{2} \overline{\mathrm{T}}}{2 \pi} \\
\mathrm{T}^{*}{ }_{M}=\overline{\mathrm{T}} \sqrt{2}
\end{gathered}
$$

Au total, la courbe type correspondant au spectre de Neuman :

a) Présente un maximum légèrement supérieur à celui observé à Chausey, pour une période nettement supérieure à celle obtenue à Chausey $(\sqrt{2} \overline{\mathrm{T}}$ au lieu de $1,25 \overline{\mathrm{T}})$;

b) Pour une période inférieure à $\overline{\mathrm{T}}$, elle est située légèrement en dessous de la courbe de Chausey, pour une période supérieure à $1,25 \overline{\mathrm{T}}$ nettement au-dessus.

Nous donnerons ci-dessous une explication possible de la différence observée pour les grandes périodes. 


\section{III. - SPECTRES ETABLIS A PRIORI SUR DONNEES METEOROLOGIQUES}

Nous avons donné ci-dessus une description simple d'une méthode permettant d'obtenir en définitive, pour un lieu d'observation donné, un nombre aussi réduit que possible de « spectres types ».

Chaque spectre de la collection possédée d'une part est rattaché à une famille, donc caractérisé par le spectre type $y^{*} \sigma(x)$ de cette famille, d'autre part dépend de deux grandeurs physiques mesurables sur l'enregistrement: $\overline{\mathrm{H}}$ et $\overline{\mathrm{T}}$.

Nous avons pu jusqu'ici nous appuyer sur les études effectuées à partir des enregistrements de Chausey; nous nous proposons maintenant d'indiquer dans quel sens pourrait s'orienter les études ultérieures.

Le premier problème à étudier est celui d'un critère permettant de diagnostiquer a priori à quelle famille se rattachera le spectre d'énergie d'un enregistrement effectué mais non encore dépouillé. Si ce diagnostic est exact, si l'on a choisi par exemple le spectre $y^{*} \sigma$, on établira :

a) Le spectre de densité spectrale, et plus précisément la zone d'incertitude $\left(W_{p}, \varepsilon\right)$;

b) Le spectre d'énergie a priori donné par les relations :

$$
k=\frac{2 \pi}{x \overline{\mathrm{T}}} \quad \mathrm{W}=\frac{\overline{\mathrm{H}}^{2} \overline{\mathrm{T}}}{2 \pi} \cdot y^{*} \sigma
$$

Si le critère de diagnostic est exact, ce spectre doit être tout entier compris dans $\left(W_{p}, \varepsilon\right)$.

Ce but étant fixé, resterait à étudier ce critìre; nous nous bornerons à indiquer quelques voies : hypotheses possibles s'excluant ou non.

a) Ce critère repose sur un examen visuel de l'état de la mer;

b) Ce critère repose sur un examen visuel de l'enregistrement $\zeta(t)$;

c) Ce critère repose sur une étude de la distribution des grandeurs apparentes $(H, T)_{j}$.

Le deuxième problème à étudier est celui de la « prévision des spectres à partir des différents facteurs physicues pouvant jouer un rôle dans la formation de la houle $\gg$.

La diversité des spectres types provient vraisemblablement d'une part de l'influence des données météorologiques, et d'autre part des conditions géographiques et océanographiques locales: le fond, le tracé des côtes, la proximité des côtes dans telle ou telle direction, etc... En particulier, il semble possible d'attribuer à la profondeur réduite au fond de la baie du Mont-Saint-Michel le fait que l'énergie observée dans les longues périodes est plus faible que ne l'indique le spectre de Neuman.

Il va de soi que le «calalogue » des spectres types doit être établi pour chaque point d'observation : par exemple celui correspondant au nord de Chausey n'est pas celui établi au sud de Chausey - du moins a priori.

Pour Neuman, les deux parametres $\bar{H}$ et $\bar{T}$ dépendent uniquement de la vitesse $v$ du vent :

$$
\frac{\mathrm{H}^{*}}{v^{5 / 2}}=\mathrm{C}^{\mathrm{t} t} \quad \frac{\overline{\mathrm{T}}}{v}=\mathrm{C}^{\mathrm{te}}
$$

à condition que celui-ci ait soufllé pendant un temps et sur une surface suffisamment longs pour que la mer ait pu atteindre son état de plein dèveloppement.

En reprenant ces notions et en les adaptant à la méthode empirique décrite plus haut, il faut rattacher par des lois qualilatives ou quantitatives $y^{*} \sigma, \overline{\mathrm{H}}, \overline{\mathrm{T}}$ aux données météorologiques :

1) actuelles locales,

2) passées locales,

3) passées lointaines.

Par exemple, dans le cas de Chausey, il faut considérer :

1) La vitesse du vent en Manche occidentale;

2) La variation du vent en Manche occidentale au cours des 24 heures, par exemple, écoulées;

3) L'existence et le déplacement des dépressions au large des còtes de France et d'Irlande dans les 48 heures écoulées.

Car il existe essentiellement à Chausey deux types de houle distincts :

- l'une d'origine océanique pénétrant entre Ouessant et Land's End,

- l'autre d'origine proche se formant dans la zone Ouessant, Land's End, Portland, Bréhat.

Lorsque les lois empiriques sont ébauchées, il est aisé de confronter les spectres prévus aux 
spectres réellement obtenus, et naturellement corriger selon les résultats les lois liant $y^{*} \sigma, \overline{\mathrm{H}}, \overline{\mathrm{T}}$ aux données météorologiques.

Lorsque ces lois seront bien au point, on pourra enfin étudier la répartition statistique annuelle des spectres d'énergie; étude qui, à notre sens, couronnerait l'ensemble des travaux effectués au site considéré.

Pour cela, il faudra répartir les différentes situations météorologiques en un certain nombre de groupes, définir pour chaque groupe une situation météorologique moyenne, et connaître enfin la répartition dans l'année moyenne de ces différentes situations météorologiques; à chaque situation, nous ferons correspondre un spectre d'énergie.

Admettons par exemple qu'au point considéré le vent local actuel influe seul sur la houle. Nous disposons d'un tableau donnant pour l'année moyenne la fréquence du vent - c'est-à-dire le nombre $n$ d'heures où ce vent souffle - en fonction de :

sa direction,

A chacun de ces vecteurs vents, l'étude de la prévision permet de rattacher un spectre d'énergie dont nous connaissons la probabilité d'apparition $\bar{\sigma}=n / 8760$.

\section{IV. - CONCLUSION}

La méthode décrite dans cet article présente l'indiscutable inconvénient d'être longue pour aboutir à son terme, et de n'être possible que pour des positions favorables où les spectres types sont en très petit nombre et où les conditions météorologiques influant sur l'état de la mer sont claires et précises.

La méthode décrite présente des avantages certains sur les méthodes classiques : elle s'adapte naturellement à toute région - sous la réserve faite ci-dessus - où l'on peut relever aisément des enregistrements de houle, elle suit pas à pas et de près dans chaque étape la réalité physique concrète.

Rappelons pour finir ses différentes étapes :

1. Dépouillements _ en laboratoire d'étude par la méthode de Tukey — d'un nombre aussi grand que possible (au minimum une trentaine) de courbes $\zeta(t)$ établies dans des conditions variées.
Etablissement des surfaces $\left(\mathrm{W}_{p i}, \varepsilon_{i}\right)$.

2. Classement de la collection obtenue en un nombre aussi restreint que possible de spectres types $y^{* \sigma}(x)$.

Etude d'un critère permettant de diagnostiquer a priori la famille $(\tau)$ à laquelle se rattachera le spectre tiré d'un enregistrement nouveau.

3. Détermination des lois empiriques, quantitatives ou qualitatives, liant les trois « paramètres $\gg: \overline{\mathrm{H}}, \overline{\mathrm{T}}, y^{*} \sigma$ aux données météorologiques.

Confrontation entre les « spectres du lendemain $\gg$ et les spectres réellement obtenus.

4. Détermination de la statistique annuelle des conditions météorologiques utilisées en "3".

Etablissement de la statistique des spectres.

\section{I S C U S I I N}

Président : M. Chapouthien

M. le Président remercic M. GoHIs de sa contribution à ce problème de mise en ordre des résultats d'une expérience. C'est le plus sonvent sous la forme spectrale qu'on conserve de la houle le souvenir le plus parlant et le plus utilisable.

M. le Président souligne le caractère statistique et non physique de la stationnarité à laquelle M. GoHn a fait allusion : il s'agit de houles progressives au sens de la physique, dont on recherche le caractère stationnaire de la période moyenne, et la méthode de Tukey ne s'applique qu'ì cette condition.

M. Miche souligne que la méthode de Tukey pour l'analyse spectrale des houles, qui représente le mode d'application pratique de la méthode inaugurée par Taylor pour la turbulence stationnaire, a fourni des résultats nettement plus satisfaisants que l'analyse harmonique habituelle, même portant sur un grand nombre de termes. Au point de vue théorique, cette dernière 
analyse, qui suppose le phẻnomène se reproduisant indéfiniment avec une période égale à la durée de l'enregistrement étudié, n'est pas conforme à la rẻalité, mais une critique analogue peut être faite à la méthode de Taylor qui suppose nulle la fonction à analyser hors du domaine étudié. C'est pourquoi il est nécessaire d'utiliser des enregistrements de houle de plusieurs minutes au moins pour neutraliser cette cause d'erreur. Par ailleurs, les enregistrements ne pourraient être trop longs (plus d'une heure par exemple), car alors l'évolution de l'agitation de la mer (modification de la période et de l'amplitude moyennes) devient appréciable.

M. GoHin indique que, pour les seiches, on peut porter l'intervalle de temps à plusieurs heures, mais que pour la houle quelques dizaines de minutes suffisent.

M. le Président conclut qu'il faut prendre la plus grande durée possible compatible avec une certaine stationnarité.

M. Мiche indique qu'il serait intéressant de comparer les spectres obtenus à des spectres théoriques, notamment à celui de Neumann (1) qui est mis à la base de la théoric de prédiction des houles de Pierson aux EtatsUnis. Il précise que cette comparaison, qu'il a pu faire à l'occasion de quelques enregistrements de houles relevés dans la Manche dans la même région (entre les fles Chausey et la pointe du Grouin) et qui ont été mis obligeamment à sa disposition par le Service d'études sur l'utilisation des marées, a révélé quelques faits intéressants.

Lanatyse de deux fortes houles lors d'une tempête de décembre 1958 a montré, en accord avec les conditions météorologiques à courte et grande distance, que l'agilation risultait de la conjonction de deux trains de houle, l'un' d'origine lointaine, l'autre proche, chacun justiciable, au moins a titre approché ef quant à la forme de son spectre, de la courbe théorique de Neumann. On peut se demander si de telles conditions ne sont pas relativement fréquentes en cas de forte houle, dans la Manche du moins.

(1) M. Gohin nous a fait remarquer, après la séance, qu'il n'a pas pu, faute de temps, indiquer à M. Mighe au cours de la discussion :

$1^{\text {a }}$ que le texte de son mémoire précise que les spectres types à une bosse ont été rapprochés du spectre de Neumann réduit;

2" qu'il estime également possible d'observer des spectres « à deux bosses » pour lesquels la méthode de spectres-types exposée ne s'applique pas telle qu'elle est actuellement. L'adaptation indispensable est en cours d'étude.
M. Banal confirme que les spectres obtenus par la méthode de Tukey ont été comparés aux spectres de Neumann.

Aucun des spectres obtenus ne faisait apparaître clairement que la houle était la composition de deux phénomènes d'origines différentes; en particulier, tous les spectres étaient à une seule bosse.

M. Baval, pense d'ailleurs qu'il faudrait que les énergies des deux trains de houle soient comparables et leurs périodes moyennes très différentes pour que le spectre de Tulsey présente deux maxima. Ces circonstances ne doivent se rencontrer en pratique que très rarement.

C'est peut-être l'inconvénient de la métlode : en essayant de réduire ce qui est fortuit, on risque de perdre une partie de l'information.

En remarque à une observation de $M$. Banal sur la forme apparente des spectres, M. Miche précise que ceux relatifs à la tempête de décembre 1958 ne présentaient qu'un seul maximum, mais trop aplati pour pouvoir s'inscrire, avec une probabilité acceptable, dans une seule courbe de Neumann.

Pour en revenir à Neumann, M. GoHis indique que : d'une part, la concordance entre le spectre réduit de Neumanu et le spectre-type déterminé à partir des curegistrements jusqu'ici dépouillés est satisfaisante. D'autre part, il lui semble délicat de chercher à rapprocher Ie spectre de Neumann non réduit, donc sous sa forme donnée par Neumañn, des spectres de Chausey nou réduits pour déterminer par exemple l'influence du vent. En effet on trouve en appliquant des formules très simples que la hauteur moyennc varie comme la puissance $5 / 2$ du vent, ce qui est certainement une puissance trop forte. En particulier pour un vent d'environ 50 noeuds, on obtient $\mathrm{H}_{1 / 3}{ }^{(6,0)}$ (amplitude de l'onde significative) de l'ordre de 25 mètres, pour 60 nouds $H_{1 / 3}^{(n) 1)}=36 \mathrm{~m}$, valeurs nettement trop fortes.

M. Miche pense que la houle, au moins dans la Manche, représente certes un phénomène aléatoire, mais souvent assez hẻtérogène. A titre d'exemple, la dénivellation de la surface de l'eau obéit bien, dans l'ensemble, à la loi de Laplace-Gauss, mais des écarts appréciables subsistent la plupart du temps. C'est pourquoi Ia méthode d'analyse de Taylor est précieuse, car elle demeure valable en toule hypothèse; elle fait ressortir l'aspect énergétique intrinsèque du phénomène. Notamment, les phases des nouvements élémentaires peuvent être quelconques et non pas nécessairement réparties au hasard. Elles pourraient varier systématiquement, on fonction des constantes du vent, par exemple; le spectre obtenu resterait identique. 